\title{
Dendritic cells combined with doxorubicin induces immunogenic cell death and exhibits antitumor effects for osteosarcoma
}

\author{
MASANORI KAWANO, KAZUHIRO TANAKA, ICHIRO ITONAGA, TATSUYA IWASAKI, \\ MASASHI MIYAZAKI, SHINICHI IKEDA and HIROSHI TSUMURA
}

Department of Orthopaedic Surgery, Faculty of Medicine, Oita University, Yufu, Oita 879-5593, Japan

Received December 5, 2014; Accepted January 13, 2016

DOI: $10.3892 /$ ol.2016.4175

\begin{abstract}
The effects of dendritic cells (DCs) with low dose doxorubicin on the enhancement of the systemic immune response, including the effects on calreticulin (CRT) expression, heat shock protein 70 (HSP70) on the cell surface expression, and the enhancement of high mobility group box 1 (HMGB1) release from cancer cells, remain unclear. The present study investigated whether the combination of DCs and doxorubicin (ADM) induces immune cell death, and leads to tumor growth inhibition in a murine osteosarcoma model. To evaluate immune response activation in vivo, 4 groups of mice were established: i) untreated mice, ii) DC-treated mice, iii) ADM-treated mice, and iv) DC and ADM-treated mice. Immunological cell death and CRT, HSP70, and HMGB1 expression levels were higher in doxorubicin-treated cells than those in untreated or those treated with DCs alone. $\mathrm{NF}-\kappa \mathrm{B}$ expression was higher in the DCs after ligand activation using CRT, HSP70, or HMGB1 in vitro. Mice treated with DCs and ADM displayed an increased number of $\mathrm{CD}^{+} \mathrm{T}$-lymphocytes within metastatic tumors and inhibition of metastatic growth. The expression of CRT and the release of HMGB1 from tumor tissues were increased in the ADM-treated groups. Treatment with DCs and $\mathrm{ADM}$ resulted in the highest serum interferon- $\gamma$ levels. Combining ADM, which can induce immunogenic cell death, with DCs enhanced the systemic immune response. The findings of the present study provide further support for the continued development of antitumor agents that induce cell death and the immune response to target osteosarcoma.
\end{abstract}

Correspondence to: Dr Masanori Kawano, Department of Orthopaedic Surgery, Faculty of Medicine, Oita University, 1-1 Idaigaoka, Yufu, Oita 879-5593, Japan

E-mail: kawano@oita-u.ac.jp

Key words: doxorubicin, immunogenic cell death, calreticulin, heat shock protein 70 , high mobility group box 1 , dendritic cell, osteosarcoma

\section{Introduction}

Osteosarcoma is the most common primary malignant bone tumor, and remarkable advances have been made in its treatment over the past several decades (1). The standard treatment for metastatic osteosarcoma is systemic combination chemotherapeutics (2); however, it would be beneficial if cancer patients could elicit tumor-specific immunity that would control or slow the growth of residual tumor cells. A combination of chemotherapy and immunotherapeutic strategies represents a challenging task, because chemotherapy is generally considered to be immunosuppressive (3).

The majority of chemotherapeutic agents induce tumor cell death by apoptosis, a process that has long been regarded as non-immunogenic (4). However, recent evidence indicates that certain anticancer drugs, such as anthracyclines, induce an immunogenic type of apoptosis that stimulates the engulfment of apoptotic bodies by dendritic cells (DCs) and the activation of cytotoxic $\mathrm{CD}^{+} \mathrm{T}$ cells through cross-priming (5). Anthracyclines serve major roles in the treatment of leukemia, lymphoma, breast, uterine, ovarian malignancies and sarcoma. Despite its side effects, doxorubicin (ADM) induces immunogenic cell death (ICD) in mouse tumor cells (6). Within hours after the initiation of ICD, pre-apoptotic tumor cells translocate calreticulin (CRT) and heat shock protein (HSP)70 from the endoplasmic reticulum to the cell surface, together with other molecules (7). One critical feature of DCs that is required to induce efficient antitumor response is the capacity to cross-present tumor-associated antigens (Ag) and to cross-prime cytotoxic $\mathrm{T}$ cells, which is a process requiring appropriate activation. In addition, cells release the late apoptosis marker high mobility group box 1 (HMGB1) into the extracellular matrix; HMGB1 can bind Toll-like receptors (8). The release of this protein appears to be required for optimal presentation of antigens from dying tumor cells, T-cell priming by DCs, and subsequent T-cell-mediated elimination of the tumor.

The present study therefore hypothesized that the antitumor effect of chemotherapeutics may be enhanced by the induction of ICD through the activation of cytotoxic T-lymphocytes (CTLs) by DCs. The aim of the present study was to investigate whether DCs enhance cell-mediated immunity in combination with ADM, and in turn induce the expression of CRT and HSP70 on the apoptotic cell surface and release HMGB1, and 
whether the combination of immunotherapy with anticancer agents has an antitumor effect in osteosarcoma mouse models.

\section{Materials and methods}

Cell lines. LM8 cells, a murine osteosarcoma line, derived from Dunn osteosarcoma, were provided by the Riken BioResource Center (Saitama, Japan). The cells were maintained in complete medium consisting of RPMI 1640 (Sigma-Aldrich, Tokyo, Japan) supplemented with 10\% heat-inactivated fetal bovine serum (Wako Pure Chemical Industries, Ltd., Osaka, Japan), $100 \mu \mathrm{g} / \mathrm{ml}$ streptomycin (Sigma-Aldrich), and $100 \mathrm{U} / \mathrm{ml}$ penicillin (Sigma-Aldrich). The cells were cultured at $37^{\circ} \mathrm{C}$ in $5 \% \mathrm{CO}_{2}$.

Immunofluorescence in cultured cells. LM8 osteosarcoma cells were seeded at a density of $1 \times 10^{5}$ cells/slide on LAB-TEK II Chamber Slides (Thermo Fisher Scientific, Inc., Waltham, MA, USA) and fixed with $4 \%$ paraformaldehyde (Wako Pure Chemical Industries, Ltd.). For immunofluorescent staining of membrane CRT and HSP70, cells were blocked with 10\% BSA (Wako Pure Chemical Industries, Ltd.) in PBS, and incubated with rabbit anti-mouse polyclonal CRT and monoclonal HSP70 antibodies (catalog no., ab2907 and ab181606; Abcam, Tokyo, Japan; 1:500 diluted in PBS with 1.5\% BSA) followed by FITC-labeled donkey anti-rabbit polyclonal immunoglobulin (Ig)G secondary antibody [ab6798; Abcam; 1:500 diluted in PBS with $1.5 \%$ goat serum (Sigma-Aldrich)]. Digital images were captured using a BIOREVO microscope equipped with a confocal microscopy system (BZ-9000, Keyence, Japan).

HMGB1 measurement. Quantification of HMGB1 in the supernatants was assessed by Quantikine ${ }^{\circledast}$ (R\&D Systems, Inc., Minneapolis, MN, USA) according to the manufacturer's protocol using Skanlt software for Multiskan ${ }^{\mathrm{TM}}$ FC plate reader (Thermo Fisher Scientific, Inc.).

Membrane and subcellular fractionation and immunoblot analysis. Membrane, cytoplasmic, and nuclear fractions were extracted using a Global Protein Fractionation kit, according to the manufacturer's instructions (St. Louis Biosciences, St. Louis, MO, USA), with minor modifications. Briefly, two cytoplasmic and nuclear fractions were extracted using NE-PER Nuclear and Cytoplasmic Extraction Reagents (Pierce $^{\mathrm{TM}}$; Thermo Fisher Scientific, Inc.). Protein $(15 \mu \mathrm{g})$ was resolved on a precast $10 \%$ Tris- $\mathrm{HCl}$ Criterion 10 -well gel (Bio-Rad Laboratories, Inc., Hercules, CA, USA) at $200 \mathrm{~V}$ (300 mAmp) for $30 \mathrm{~min}$. The gel was wet-transferred to a PVDF membrane for $1 \mathrm{~h}$, and blocked with PBST containing $5 \%$ instant dry non-fat milk for $30 \mathrm{~min}$ at room temperature. The following antibodies were obtained from Cell Signal Technology (Danvers, MA, USA): Rabbit monclonal anti-mouse CRT (catalog no., 12238), polyclonal HSP70 (catalog no., 4876), polyclonal HMGB1 (catalog no., 3935), monclonal IgG NF- $\kappa$ B (catalog no., 3017), polyclonal IкB- $\alpha$ (catalog no., 9242) and polyclonal $\alpha$-Tubulin (catalog no., 2144). The antibodies were diluted at 1:1000 and were incubated for $1 \mathrm{~h}$ at room temperature. Immunocomplexes were visualized with horseradish peroxidase-conjugated anti-rabbit $\operatorname{IgG}$ antibodies (GE Healthcare, Tokyo, Japan), developed the blots using
ECL Plus system (GE Healthcare) with a ChemiDoc camera (ImageQuant LAS 4000mini; GE Healthcare). The quantification of western blot signals was performed by the densitometry with ImageQuant TL version 8.1 software (GE Healthcare). All western blot experiments were repeated at least three times.

Animals. A total of $1 \times 10^{6}$ LM8 cells were hypodermically implanted into the subcutaneous gluteal region of 24 female $\mathrm{C} 3 \mathrm{H}$ mice 6-8 weeks old. The $\mathrm{C} 3 \mathrm{H}$ mice were purchased from Sankyo Labo, Inc. (Toyama, Japan) and housed in a specific pathogen-free animal facility at Oita University (Oita, Japan).

The animal experimental protocol was approved by the Ethics Review Committee for Animal Experimentation of Oita University (Oita, Japan), and all mice used in the present study were anesthetized with ketamine/xylazine or isoflurane/oxygen for experiments and euthanized with cervical dislocation under anesthesia. All efforts were made to minimize suffering.

Generation of DCs. Bone marrow-derived DCs were generated as described by Lutz and Rössner (9) with minor modifications. Briefly,erythrocyte-depleted mouse bone marrow cells obtained from flushed femur bone marrow cavities $\left(1 \times 10^{6}\right.$ cells $\left./ \mathrm{ml}\right)$ were cultured in complete medium with $20 \mathrm{ng} / \mathrm{ml}$ granulocyte macrophage colony-stimulating factor (GM-CSF; PeproTech EC Ltd., London, UK), recombinant mouse GM-CSF (100 ng/ml; PeproTech EC Ltd.) and IL-4 (R\&D Systems Europe, Ltd., Abingdon, UK) at $25 \mathrm{ng} / \mathrm{ml} \mathrm{(U/ml)} \mathrm{in} 10-\mathrm{cm}$ tissue culture dishes at $37^{\circ} \mathrm{C}$ in an atmosphere containing in $5 \% \mathrm{CO}_{2}$.

Flow cytometry. For flow cytometric analysis,DCs were counted with a FACSVerse ${ }^{\mathrm{TM}}$ Flow Cytometer (Becton Dickinson, San Jose, CA, USA) and stained with fluorochrome-conjugated antibodies (BD Pharmingen ${ }^{\mathrm{TM}}$; BD Biosciences, Tokyo, Japan) for the following markers: APC hamster anti-mouse monoclonal cluster of differentiation (CD)11c (catalog no., 550261), PE hamster anti-mouse monoclonal CD80 (catalog no., 553769) and APC rat anti-mouse monoclonal CD86 (catalog no., 558703). The antibodies diluted at 1:400 and were stained for $1 \mathrm{~h}$ at room temperature. CD11c was used as a marker for all DCs regardless of the degree of maturation, whereas CD80, CD86 are markers for DCs. Data analysis was performed with FACSuite $^{\mathrm{TM}}$ version 1.0.3 software (Becton Dickinson).

Therapy protocol. All the animals developed tumors. The maturation of the DCs were assessed at day 4 (Fig. 1A) and day 7 (Fig. 1B) using flow cytometry. The following 4 groups were established (Fig. 1C): i) Untreated (control; n=6); ii) DCs were injected twice a week into the subcutaneous contralateral gluteal region (DC; $n=6)$; iii) intraperitoneal injection of ADM (ADM; $6 \mathrm{mg} / \mathrm{kg}$ of body weight; $\mathrm{n}=6$ ); and (iv) DCs were injected twice a week into the subcutaneous contralateral gluteal region and intraperitoneal injection of ADM $(6 \mathrm{mg} / \mathrm{kg}$ of body weight) was performed twice per week (DC+ADM; $\mathrm{n}=6$ ). All experiments were performed under the guidelines for animal experiments as stipulated by the Oita University Graduate School of Medical Science.

Tumor volume. Tumor volumes were measured using the micro-CT apparatus (R_mCT) which allows us to obtain 
A

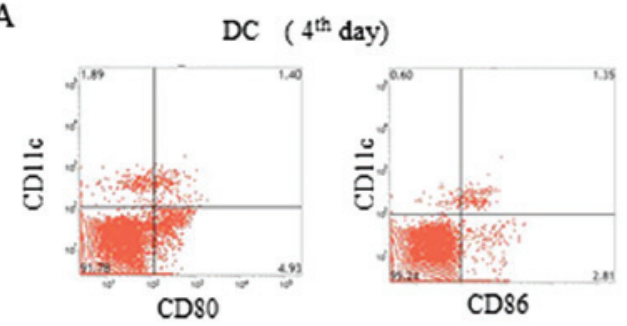

B

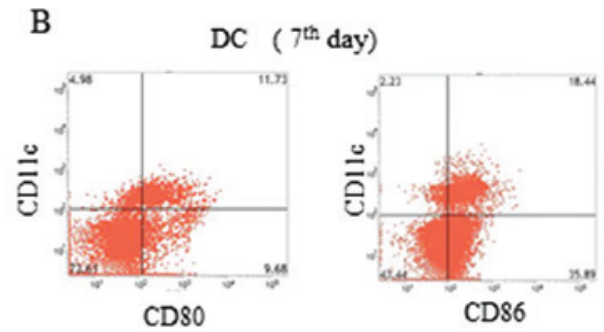

C

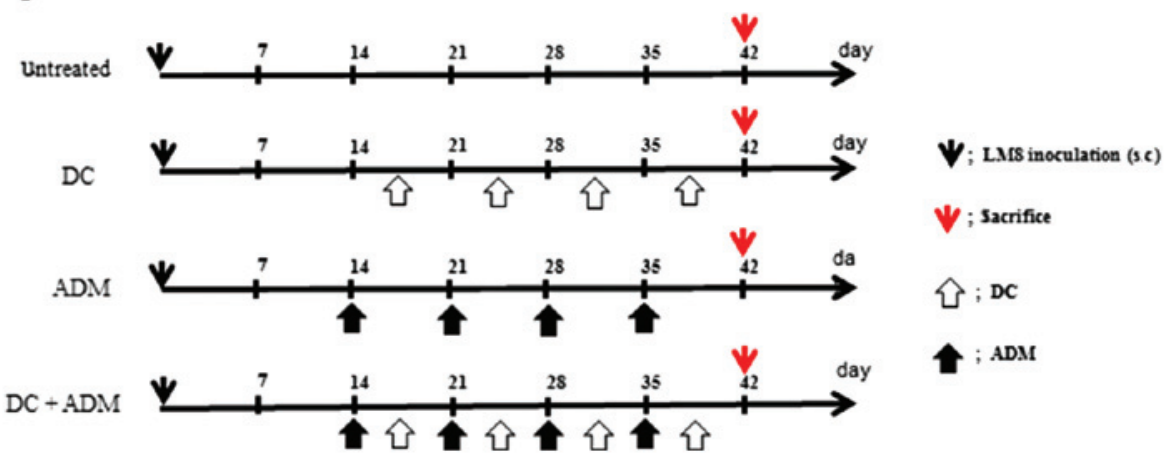

Figure 1. (A) DC maturation status examined using flow cytometry. DCs at culture day 7 were more mature than DCs at culture day 4. (B) A diagram of the experimental protocol and treatment schedule. (C) Four groups of mice: untreated mice, DC-treated mice, ADM-treated mice, and DC+ADM-treated mice. DC, dendritic cell; ADM, doxorubicin.

high-resolution CT images in small living animals. The I-view-R (J. Morita Mfg Corp, Kyoto, Japan) was used as the viewer, and diagnosis was made with slice images viewed in all directions. Tumor volumes were estimated using the following formula: Tumor volume $=\left(\right.$ length $\mathrm{x}$ width $\left.{ }^{2}\right) / 2$.

Immunohistochemistry. Immunohistochemistry was used to measure the levels of CD11c, as a marker of DCs; CD8, as a marker of cytotoxic T lymphocytes; and CRT and HMGB1, inside tumor lesions. Primary tumor lesions were resected and all the samples were fixed in a frozen section. Six samples per mouse were cut into $10-\mu$ m-thick slices. The tissue sections were incubated overnight at room temperature with primary rabbit anti-mouse polyclonal CD8- $\alpha$ (catalog no., sc-7188; Santa Cruz Biotechnology, Inc., Dallas, TX, USA), monoclonal CD11c (catalog no., ab52632; Abcam), polyclonal CRT, monoclonal HSP70 and monoclonal HMGB1 (catalog no., ab79823; Abcam), which were diluted at 1:200 in Ab Diluent (Dako ChemMate, Dako, Japan). The secondary antibody for CD11c and CRT was FITC-labeled donkey anti-rabbit polyclonal IgG (catalog no., A-21206; Thermo Fisher Scientific, Inc.) and for CD8, HSP70 and HMGB1 was Rhodamine red-labeled goat anti-rabbit polyclonal IgG (catalog no., R-6394; Thermo Fisher Scientific, Inc.). The secondary antibodies were diluted at 1:300 in $\mathrm{Ab}$ diluent and added for $60 \mathrm{~min}$ at room temperature in the dark. Digital images were captured using BIOREVO microscope equipped with a confocal microscopy system (BZ-9000).

Cytokine evaluations. Serum interferon (IFN)- $\gamma$ and interleukin (IL)-12 release in mice were measured by enzyme-linked immunosorbent assay (ELISA) using Quantikine ${ }^{\circledR}$ (R\&D Systems, Inc.) according to the manufacturer's protocol and a Skanlt for Multiskan FC plate reader.
Statistical analysis. The difference among the four groups was determined using a non-repeated measures analysis of variance and the Scheffe test. All analyses were conducted using SPSS 18.0 software (SPSS, Inc., Chicago, IL, USA). Results were expressed as the mean \pm standard deviation, and $\mathrm{P}<0.05$ was considered to indicate a statistically significant difference.

\section{Results}

ADM induced ICD in LM8 cells in vitro. To verify the ability of ADM to induce ICD, the expression of CRT and HSP70 on the cell surface and the release of HMGB1 in the supernatant was evaluated by immunofluorescence. ADM treatment markedly increased the expression of CRT and HSP 70 on the cell membrane (Fig. 2A). The protein expression levels of CRT and HSP70 on the cell surface was also increased in ADM-treated cells (Fig. 2B). In addition, the amount of HMGB1 in the cell culture supernatant was significantly increased after ADM treatment alone (Fig. 2C; $\mathrm{P}=0.00043$ ).

Ligand administration induced DC activation. CRT, HSP70, and HMGB1 ligands were administered to DCs in culture to verify the ability of these ligands to activate DC function. DCs treated with CRT (Fig. 3A), HSP (Fig. 3B) and HMGB1 (Fig. 3C) and HMGB1 ligands were activated, as compared to untreated DCs, as assessed by flow cytometric analysis. Thirty minutes after CRT (Fig. 3D), HSP70 (Fig. 3E) and HMGB1 (Fig. 3F) ligand treatment, the amount of $\mathrm{NF}-\kappa \mathrm{B}$ in the nuclear fraction was higher than the amount of NF- $\kappa \mathrm{B}$ in the cytoplasmic fraction and $\mathrm{I} \kappa \mathrm{B}$ in whole lysate, as assessed by western blot analysis. Thus, CRT, HSP, and HMGB1 ligands could activate DCs function in vitro. 
A

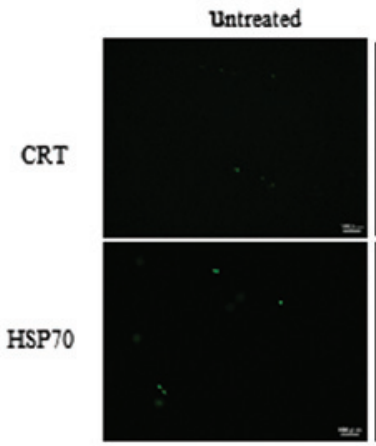

B

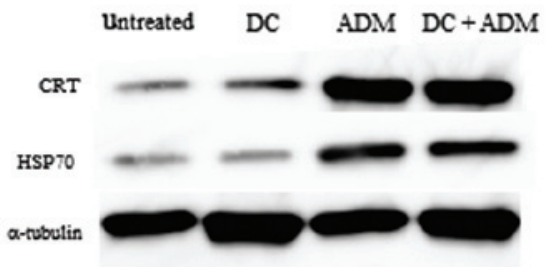

DC

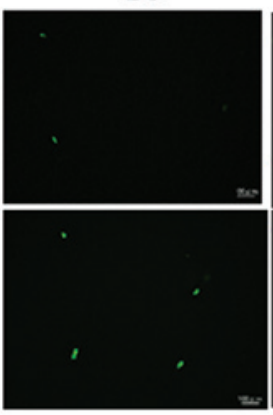

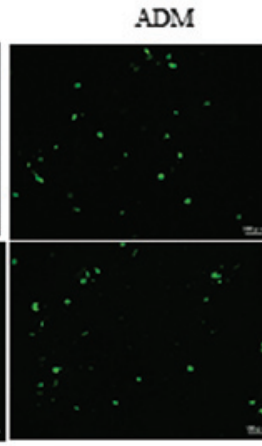

$\mathrm{DC}+\mathrm{ADM}$

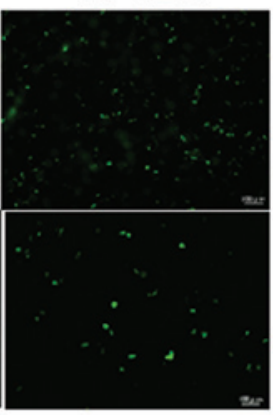

C

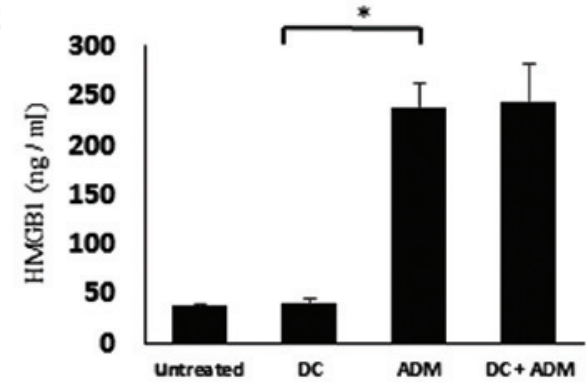

Figure 2. (A) Immunofluorescence in the four groups to detect CRT and HSP70 expression on the cell surface. (B) Immunoblotting of CRT, HSP70, and $\alpha$-tubulin on the cell membrane fraction. (C) ELISA detection of HMGB1 secretion in supernatant. CRT, calreticulin; ADM, doxorubicin; HSP70, heat shock protein 70 ; DC, dendritic cell. ${ }^{*} \mathrm{P}<0.05$.

A

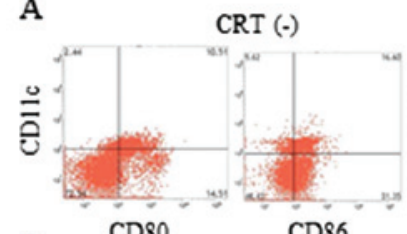

B

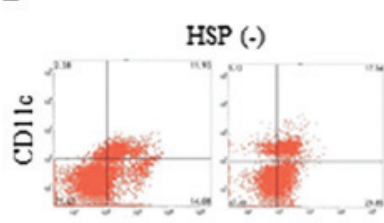

C
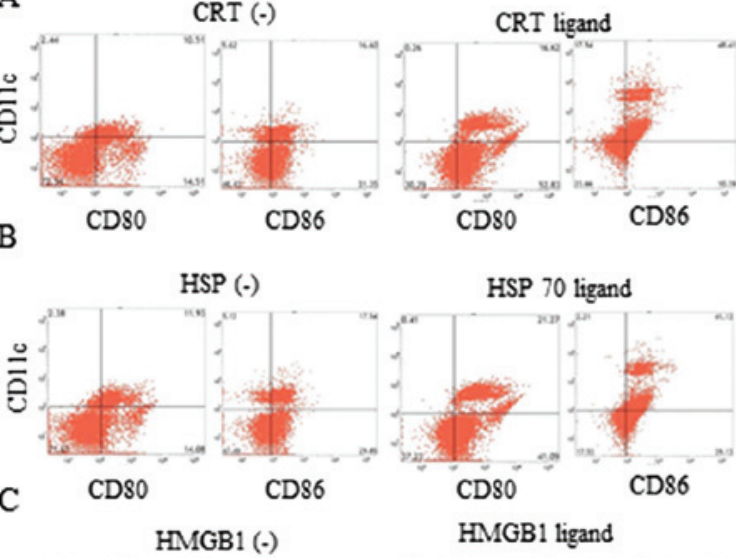

HSP 70 ligand
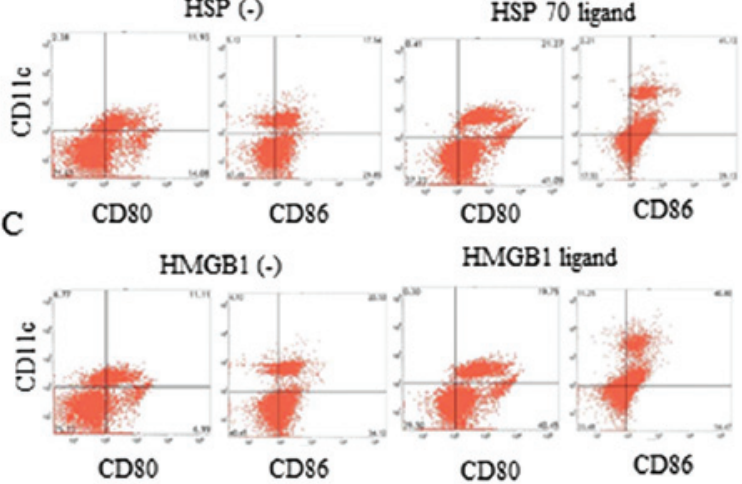

HMGB1 ligand
D

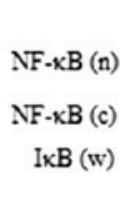

E

HSP70 ligand (+)

$\mathrm{NF}-\mathrm{xB}(\mathrm{n})$

$\mathrm{NF}-\mathrm{kB}(\mathrm{c})$

IKB (w)

F

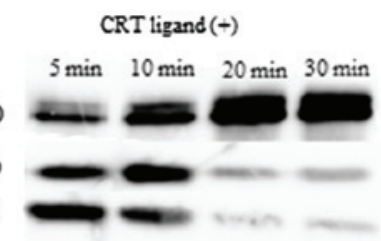

$5 \mathrm{~min} 10 \mathrm{~min} 20 \mathrm{~min} 30 \mathrm{~min}$
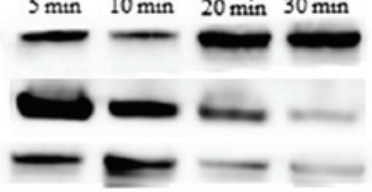

HMGBl ligand ( + )

$\mathrm{NF}-\mathrm{kB}(\mathrm{n})$

$5 \min 10 \min 20 \min 30 \mathrm{~min}$

NF- $\mathrm{xB}(\mathrm{c})$

$\mathrm{I} \times \mathrm{B}(\mathrm{w})$

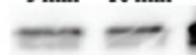

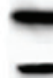

Figure 3. DC activation status examined using flow cytometry. The change in CD80 and CD86 expression on DCs with or without the (A) CRT ligand,

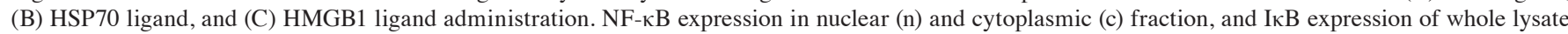
(w) up to 30 min after treatment with the (D) CRT ligand, (E) HSP70 ligand, and (F) HMGB1 ligand. DC, dendritic cell; CRT, calreticulin; ADM, doxorubicin; HSP70, heat shock protein 70; HMGB1, high mobility group box 1.

Tumor volume of the tumor lesion. A total of 42 days after the tumor cell inoculation, the volume of the metastatic lesion in mice that received a DC injection in combination with ADM treatment $\left(366.67 \pm 14.95 \mathrm{~mm}^{3}\right)$ was significantly lower $(\mathrm{P}<0.01)$ than that observed in mice that received DCs $\left(782.41 \pm 13.66 \mathrm{~mm}^{3}\right)$ or ADM alone $\left(677.69 \pm 26.01 \mathrm{~mm}^{3}\right.$; Fig. 4A).
ADM induced ICD in mice tumor tissues. The ability of ADM to induce the expression of ICD markers in tumors in vivo was then determined. Mice receiving ADM injections demonstrated a marked increase in CRT and HSP70 expression on the cell membrane (Fig. 4B). The release of HMGB1 in the tumor tissues was further analyzed. ADM induced release of HMGB1 in all tested mice (Fig. 4C). 


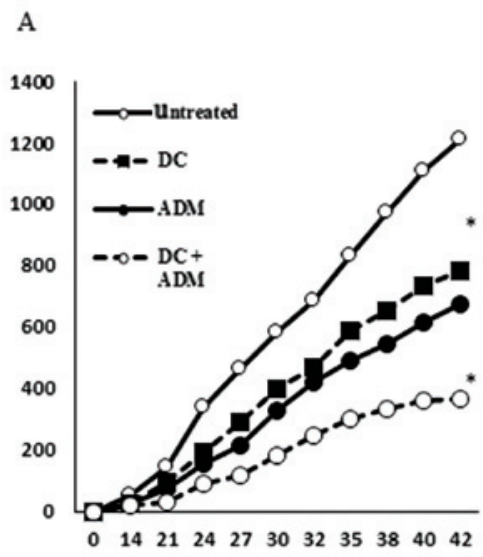

B

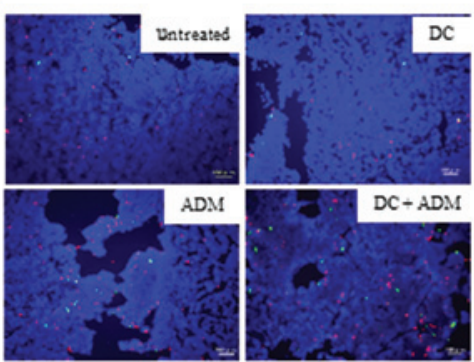

$\mathrm{C}$

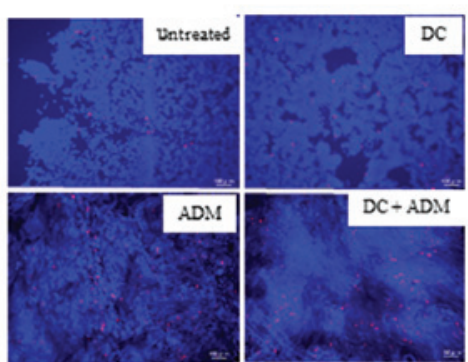

Figure 4. (A) The tumor volume of the primary lesion in the four treatment groups was evaluated using micro-CT. (B) To evaluate CRT (green) and HSP70 (red) expression in primary lesion, immunofluorescence was performed in the four groups. (C) The positive cells expressing HMGB1 (red) in the four treatment groups are shown. DC, dendritic cell; CRT, calreticulin; ADM, doxorubicin; HSP70, heat shock protein 70; HMGB1, high mobility group box 1. " P<0.05 vs. untreated.
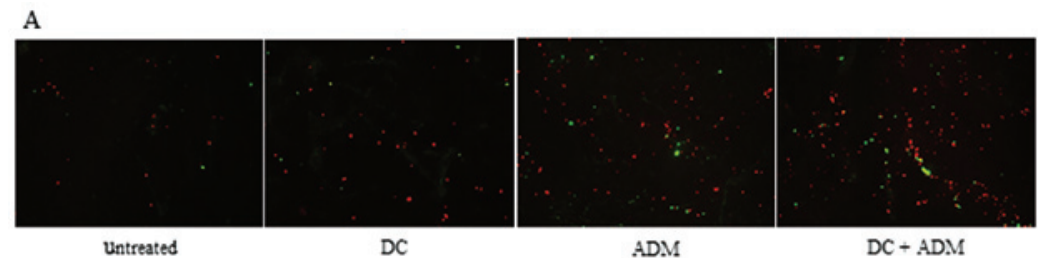

B

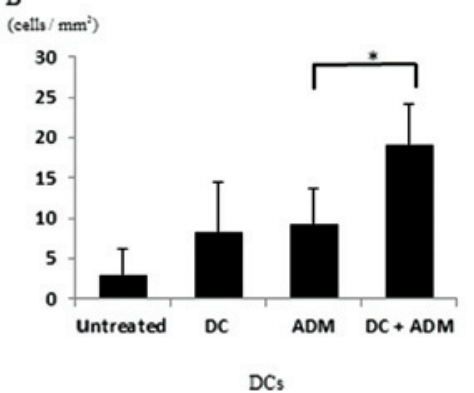

C

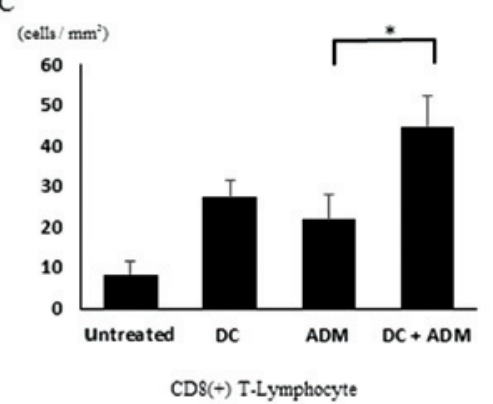

Figure 5. (A) To evaluate CD8 $8^{+}$T-lymphocytes (red) and DCs (green) in tumor tissues, immunofluorescence was performed. The number of (B) CD8 T-lymphocytes and (C) DCs per unit area in the four treatment groups are shown. DC, dendritic cells. ${ }^{*} \mathrm{P}<0.05$.

Infiltration of $C D 8^{+}$T-lymphocytes and DCs within the tumor. CD8 ${ }^{+}$T-lymphocytes gathered within the primary tumor in ADM-treated mice (Fig. 5A). DCs were also recruited to the metastatic area in the ADM-treated groups compared with the non-ADM-treated groups. The number of $\mathrm{CD}^{+} \mathrm{T}$-lymphocytes per unit area was significantly higher $(\mathrm{P}<0.01)$ in the mice that received DCs combined with ADM $\left(44.78 \pm 7.44\right.$ cells $\left./ \mathrm{mm}^{2}\right)$ than in those that received DCs $\left(27.38 \pm 4.31\right.$ cells $\left./ \mathrm{mm}^{2}\right)$ or ADM alone (21.74 \pm 6.43 cells $/ \mathrm{mm}^{2}$; Fig. 5B). The number of $\mathrm{CD} 1 \mathrm{C}^{+}$cells per unit area was significantly higher $(\mathrm{P}<0.01)$ in the mice that received DCs combined with ADM $\left(19.23 \pm 5.032\right.$ cells $\left./ \mathrm{mm}^{2}\right)$ than in those that received DCs $\left(8.34 \pm 6.22\right.$ cells $\left./ \mathrm{mm}^{2}\right)$ or ADM alone $\left(9.32 \pm 4.324\right.$ cells $/ \mathrm{mm}^{2}$; Fig. 5C).
Cytokine release. Mice treated with both DCs and ADM displayed higher serum IFN- $\gamma$ levels $(365.64 \pm 45.32 \mathrm{pg} / \mathrm{ml}$; $\mathrm{P}<0.01)$ than those received DCs $(155.53 \pm 13.01 \mathrm{pg} / \mathrm{ml})$ or ADM treatment alone $(138.37 \pm 28.11 \mathrm{pg} / \mathrm{ml}$; Fig. 6A). Mice treated with both DCs and ADM displayed higher serum IL-12 levels $(243.52 \pm 39.92 \mathrm{pg} / \mathrm{ml} ; \mathrm{P}<0.01)$ than those that received DCs $(145.43 \pm 16.38 \mathrm{pg} / \mathrm{ml})$ or ADM treatment alone $(133.72 \pm 21.29$ pg/ml; Fig. 6B).

\section{Discussion}

The majority of osteosarcoma patients are treated with a combination of surgery and chemotherapy. Despite recent 

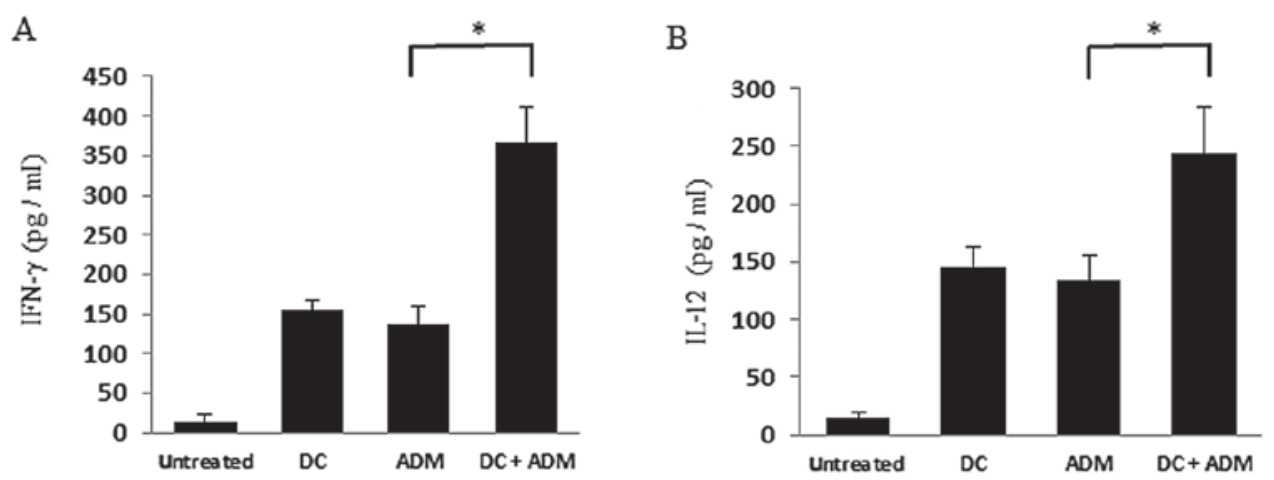

Figure 6. (A) The mice that received DCs+ADM exhibited the highest IFN- $\gamma$ levels. (B) The mice that received DCs+ADM exhibited the highest IL-12 levels. DC, dendritic cells; ADM, doxorubicin; IFN- $\gamma$, interferon- $\gamma$; IL-12, interleukin-12. ${ }^{*} \mathrm{P}<0.05$.

advances in locally, curative therapy, chemotherapeutic treatments for metastatic disease are often ineffective. Therefore, the development of novel therapeutic options is of great interest. The present authors have previously investigated the effect of immunotherapy using DCs on cell death (10); and the combination of DCs with anti-regulatory T cells (Tregs) antibodies, such as anti-transforming growth factor $\beta$ (TGF- $\beta$ ) (11) or anti-cytotoxic T-Lymphocyte Antigen 4 (CTLA-4) (12).

Certain chemotherapeutic regimens trigger cancer cell death through stimulating endogenous immune responses (13-15). Identification of potent activating signals expressed by immunogenic tumor cells would significantly contribute to understanding the interaction between tumor cells and the immune system and would facilitate the design of more effective immunotherapeutic strategies.

ICD elicited by chemotherapeutics is characterized by a series of events that include pre-apoptotic surface translocation of CRT and HSP70, and the release of HMGB1 into the extracellular milieu. These proteins can bind to DCs and enhance adaptive antitumor responses $(6,16)$. The present study initially examined whether ADM could induce ICD in a mouse osteosarcoma cell line. Increased expression of CRT and HSP70 indicated that ADM treatment may translocate immunogenic factors to the cell membrane. ADM also induced the release of HMGB1, as shown by ELISA. Further, ICD-related ligands, including CRT, HSP70 and HMGB1, were secreted in LM8 cells, at least in these culture conditions.

In the present study, the interaction between immature DCs and immunogenic tumor cells led to increased tumor cell uptake and induced moderate expression of maturation-associated markers on DCs, such as CD80 and CD86. Similar to published studies by Zitvogel et al (15), the rate of phagocytosis in the present study correlated with the intensity of CRT expression and, to a lesser degree, with HSP expression (17). In addition, DC activation through ligand ligation can be evaluated through $\mathrm{NK}-\kappa \mathrm{B}$ expression in the nucleus (18). A higher percentage of mature DCs was observed when DCs were cocultured with ADM-treated LM8 cells. NF- $\mathrm{BB}$ expression in the DC nuclear fraction was increased, and cytoplasmic $\mathrm{NF}-\kappa \mathrm{B}$ and I $\mathrm{B}$ significantly decreased within $30 \mathrm{~min}$. Thus, one may conclude that ICD-related ligands induced DC activation in the present study.

The effect of ADM-induced ICD in vivo was analzed in the present study using a mouse model of osteosarcoma. Normal mice with tumors that formed from injected LM8 osteosarcoma cells were treated with DCs generated from bone marrow, and ADM was administered. CRT and HSP70 translocation to the cell surface and HMGB1 release was observed after ADM treatment. Combination treatment with DCs and ADM resulted in smaller lesions. Thus, ADM administered via the tail vein induced ICD in the established tumors tissues, and DCs may cooperate at the tumor site through activation by ICD ligands.

Upon exposure to ADM, the expression of CRT and HSP70 expression on the surface of dying cells may be a marker for ICD, which may deliver an activating stimulus to DCs. HMGB1 is actively secreted from inflammatory cells or released from necrotic cells $(19,20)$, and it signals through TLR2, TLR4, and RAGE in DCs $(21,22)$. DCs should migrate to the site of release, and activate to transmit information to CTLs. To evaluate whether tumor cells expressing ICD markers could recruit DCs and $\mathrm{CD}^{+} \mathrm{T}$ cell following $\mathrm{DC}$ infiltration, the ability of tumor cell-loaded DCs to activate tumor cell-specific T cell responses was further evaluated. In mice treated with DCs and ADM, the number of $\mathrm{CD}^{+} \mathrm{T}$ cells and DCs inside the tumor tissue increased compared with mice treated with DCs or ADM alone. This is consistent with the previous results demonstrating that tumor lesion volumes were significantly reduced after combination therapy. Taken together these data suggest that ICD factors may facilitate the activity of DCs and CTLs in the tumor.

DCs loaded with tumor cells killed by ADM efficiently stimulated the activation of cell-mediated immunity by increasing serum IFN- $\gamma$ and IL-12 levels. These cells also induced significantly higher secretion of cytotoxic cytokines compared with non-immunogenic tumor cells, which may be relevant for the design of cancer immunotherapy studies. The results of the present study revealed that stimulating DCs using ADM-treated tumor cells could enhance cell-mediated immunity.

Despite the continuous introduction of novel drugs and improved chemotherapy protocols, it is likely that, at some point, chemotherapy will reach its limits, and clinical efficacy will plateau. A combination of treatment modalities classically surgery with chemo- or radiotherapy has been a standard strategy for cancer treatment. Many clinical studies based on the combination of chemotherapy and immunotherapy have demonstrated variable responses (23). Indeed, chemotherapy 
may be either immunostimulatory or immunosuppressive, depending on the dosage and the timing of administration, and may synergize with immunotherapy approaches in vivo (24). Chemotherapy and immunotherapy should not be considered antagonizing forms of therapy; rather, it is conceivable that their combination could substantially improve the prognosis of cancer patients.

\section{Acknowledgements}

The authors thank Dr Hidekatsu Iha for helpful discussions regarding the present study.

\section{References}

1. Stiller CA, Bielack SS, Jundt G and Steliarova-Foucher E: Bone tumours in European children and adolescents, 1978-1997. Report from the Automated Childhood Cancer Information System project. Eur J Cancer 42: 2124-2135, 2006.

2. Bielack SS, Kempf-Bielack B, Delling G, Exner GU, Flege S, Helmke K, Kotz R, Salzer-Kuntschik M, Werner M, Winkelmann W, et al: Prognostic factors in high-grade osteosarcoma of the extremities or trunk: An analysis of 1,702 patients treated on neoadjuvant cooperative osteosarcoma study group protocols. J Clin Oncol 20: 776-790, 2002.

3. Milosević DB: The different level of immunological recovery after chemotherapy in leukemia and lymphoma patients. J Exp Clin Cancer Res 20: 517-522, 2001.

4. Tesniere A, Panaretakis T, Kepp O, Apetoh L, Ghiringhelli F, Zitvogel L and Kroemer G: Molecular characteristics of immunogenic cancer cell death. Cell Death Differ 15: 3-12, 2008.

5. Apetoh L, Mignot G, Panaretakis T, Kroemer G and Zitvogel L: Immunogenicity of anthracyclines: Moving towards more personalized medicine. Trends Mol Med 14: 141-151, 2008.

6. Apetoh L, Ghiringhelli F, Tesniere A, Obeid M, Ortiz C, Criollo A, Mignot G, Maiuri MC, Ullrich E, Saulnier P, et al: Toll-like receptor 4-dependent contribution of the immune system to anticancer chemotherapy and radiotherapy. Nature 13: 1050-1059, 2007.

7. Fucikova J, Kralikova P, Fialova A, Brtnicky T, Rob L, Bartunkova J and Spísek R: Human tumor cells killed by anthracyclines induce a tumor-specific immune response. Cancer Res 71: 4821-4833, 2011.

8. Sancho D, Joffre OP, Keller AM, Rogers NC, Martínez D, Hernanz-Falcón P, Rosewell I and Reis e Sousa C: Identification of a dendritic cell receptor that couples sensing of necrosis to immunity. Nature 458: 899-903, 2009.

9. Lutz MB and Rössner S: Factors influencing the generation of murine dendritic cells from bone marrow: The special role of fetal calf serum. Immunobiology 212: 855-862, 2007.
10. Kawano M, Nishida $H$, Nakamoto $Y$, Tsumura $H$ and Tsuchiya $H$ : Cryoimmunologic antitumor effects enhanced by dendritic cells in osteosarcoma. Clin Orthop Relat Res 468: 1373-1383, 2010.

11. Kawano M, Itonaga I, Iwasaki T, Tsuchiya $\mathrm{H}$ and Tsumura $\mathrm{H}$ : Anti-TGF- $\beta$ antibody combined with dendritic cells produce antitumor effects in osteosarcoma. Clin Orthop Relat Res 470: 2288-2294, 2012

12. Kawano M, Itonaga I, Iwasaki T and Tsumura $\mathrm{H}$ : Enhancement of antitumor immunity by combining anti-cytotoxic $\mathrm{T}$ lymphocyte antigen- 4 antibodies and cryotreated tumor lysate-pulsed dendritic cells in murine osteosarcoma. Oncol Rep 29: 1001-1006, 2013.

13. Zitvogel L, Kepp O and Kroemer G: Immune parameters affecting the efficacy of chemotherapeutic regimens. Nat Rev Clin Oncol 8: 151-160, 2011.

14. Locher C,Conforti R,Aymeric L,Ma Y, YamazakiT,RusakiewiczS, Tesnière A, Ghiringhelli F, Apetoh L, Morel Y, et al: Desirable cell death during anticancer chemotherapy. Ann N Y Acad Sci 1209: 99-108, 2010.

15. Zitvogel L, Kepp O, Senovilla L, Menger L, Chaput N and Kroemer G: Immunogenic tumor cell death for optimal anticancer therapy: The calreticulin exposure pathway. Clin Cancer Res 16: 3100-3104, 2010.

16. Obeid M, Tesniere A, Ghiringhelli F, Fimia GM, Apetoh L, Perfettini JL, Castedo M, Mignot G, Panaretakis T, Casares N, et al: Calreticulin exposure dictates the immunogenicity of cancer cell death. Nat Med 13: 54-61, 2007.

17. Michaud M, Sukkurwala AQ, Di Sano F, Zitvogel L, Kepp O and Kroemer G: Synthetic induction of immunogenic cell death by genetic stimulation of endoplasmic reticulum stress. Oncoimmunology 3: e28276, 2014.

18. Hoshino K and Kaisho T: Nucleic acid sensing toll-like receptors in dendritic cells. Curr Opin Immunol 20: 408-413, 2008.

19. Wang H, Vishnubhakat JM, Bloom O, Zhang M, Ombrellino M, Sama A and Tracey KJ: Proinflammatory cytokines (tumor necrosis factor and interleukin 1) stimulate release of high mobility group protein-1 by pituicytes. Surgery 126: 389-392, 1999.

20. Scaffidi P, Misteli T and Bianchi ME: Release of chromatin protein HMGB1 by necrotic cells triggers inflammation. Nature 418: 191-195, 2002.

21. Park JS, Svetkauskaite D, He Q, Kim JY, Strassheim D, Ishizaka A and Abraham E: Involvement of toll-like receptors 2 and 4 in cellular activation by high mobility group box 1 protein. J Biol Chem 279: 7370-7377, 2004.

22. Apetoh L, Ghiringhelli F, Tesniere A, Criollo A, Ortiz C, Lidereau R, Mariette C, Chaput N, Mira JP, Delaloge S, et al: The interaction between HMGB1 and TLR4 dictates the outcome of anticancer chemotherapy and radiotherapy. Immunol Rev 220: 47-59, 2007.

23. Rosenberg SA, Restifo NP, Yang JC, Morgan RA and Dudley ME: Adoptive cell transfer: A clinical path to effective cancer immunotherapy. Nat Rev Cancer 8: 299-308, 2008.

24. Nowak AK, Lake RA and Robinson BW: Combined chemoimmunotherapy of solid tumours: Improving vaccines? Adv Drug Deliv Rev 58: 975-990, 2006. 